\title{
Searching for Faint Exozodiacal Disks: Keck Results and LBTI Status
}

\author{
D. Defrère ${ }^{1}$, P. Hinz ${ }^{1}$, B. Mennesson ${ }^{2}$, R. Millan-Gabet ${ }^{3}$, A. Skemer ${ }^{1}$, \\ V. Bailey ${ }^{1}$ and T.J. Rodigas ${ }^{1}$ \\ ${ }^{1}$ Steward Observatory, ${ }^{2}$ Jet Propulsion Laboratory, ${ }^{3}$ Caltech \\ email: ddefrere@email.arizona.edu
}

\begin{abstract}
The possible presence of dust in the habitable zone around nearby main-sequence stars is considered as a major hurdle toward the direct imaging of Earth-like extrasolar planets with future dedicated space-based telescopes (e.g., Roberge et al. 2012). In this context, NASA has funded two ground-based mid-infrared nulling interferometers to combine the large apertures available at the Keck Observatory and the Large Binocular Telescope (LBT). In this poster, we present the preliminary results of the extended survey carried out with the Keck Interferometer Nuller (KIN) between 2008 and 2011 and describe the forthcoming LBTI survey.
\end{abstract}

Keywords. infrared, exozodiacal dust, surveys, nulling interferometry, high angular resolution

\section{Nulling interferometry}

Nulling interferometry is a technique proposed by Bracewell 35 years ago to image extra-solar planets (Bracewell 1978). The basic principle is to combine the beams coming from two distinct telescopes in phase opposition so that a dark fringe appears on the line of sight, which strongly reduces the stellar emission while transmitting the flux of off-axis sources located at angular spacings which are a multiple of $\lambda / b$ (where $b$ is the distance between the telescope and $\lambda$ the wavelength of observation). The technique has now been successfully used on sky both in the near-infrared (e.g., Mennesson et al. 2011) and the mid-infrared (e.g., Stock et al. 2010) and the performance required to directly detect exoEarths demonstrated on laboratory testbeds (e.g., Martin et al. 2012).

\section{Keck results}

Science observations with the KIN started in 2008 with four different exozodi programs and ended in 2011 with the observation of 48 stars. The results of one of these programs have been published by Millan-Gabet et al. (2011), providing unprecedented sensitivity to warm exozodiacal dust in the habitable zone of 25 nearby single main-sequence stars. The analysis of the remaining data is currently ongoing (Mennesson et al. in prep). It includes a total of 41 single stars, i.e., with no known companion within the $\sim 0.5$ " interferometric FOV, divided in three main sub-samples: (1) stars with no previous detection of dust; (2) presumably dusty stars; and (3) stars with an interferometric near-infrared detection (Absil et al. 2013). The preliminary results are shown in Fig.1. $\eta$ Crv is the only clear KIN detection although the statistical analysis of the measurements suggests that an additional $\sim 10$ objects are good candidates for a KIN excess and will be followed-up with the LBTI. 


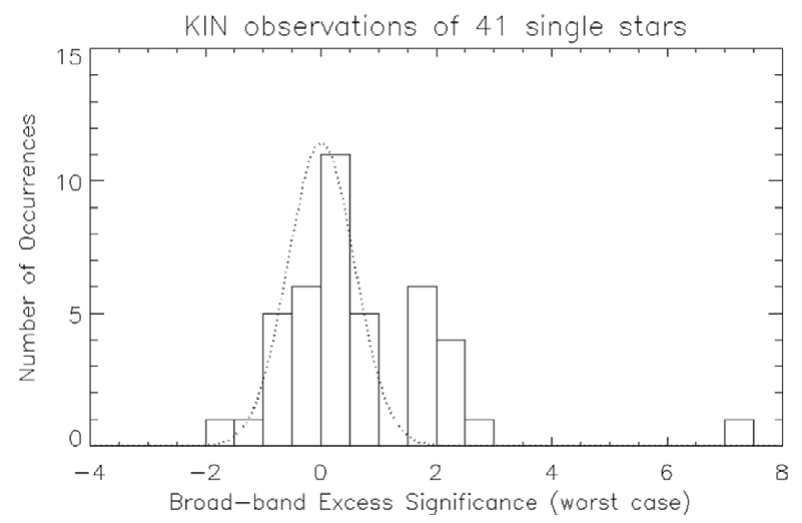

Figure 1. Histogram of broad-band (8 to 13 microns) excess "significance" measured around 41 stars of the KIN extended sample, compared to a centered Gaussian distribution representative of detection noise (dotted line, Mennesson et al. in prep).

\section{LBTI status}

The Large Binocular Telescope Interferometer (LBTI) is the ideal ground-based instrument to carry out a deep survey for exozodiacal dust around nearby main-sequence stars. It combines high sensitivity (provided by the two 8.4-m mirrors on a single structure), high contrast (see companion paper by V. Bailey on AO performance), and high resolution (equivalent to the one of a 22.7-m telescope). LBTI was installed in September 2010 at the LBT and first fringes were obtained one month later. Dual-aperture AOcorrected interferometric fringes were realized in April 2012 and first null measurements in September 2012. Phase tracking and differential tip-tilt correction are currently under commissioning.

The exozodi survey is called HOSTS (Hunt for Observable Signatures of Terrestrial Planetary Systems) and is expected to start in 2013. It is currently being defined by the LBTI Key Science Team (nexsci.caltech.edu/missions/LBTI/KeySciTeam.shtml) and currently consists in a shallow survey of 60-80 FGK nearby main-sequence stars among which 10 are well-known dusty systems and 10 are the best candidates for future exoEarth direct imaging instruments. The goals of the survey are: (1) to constrain the mean exozodiacal dust density; (2) to study exozodiacal dust disk evolution; (3) to understand the connection between exozodiacal dust and planets; and (4) to correlate exozodiacal dust with basic stellar properties or the presence of dust inferred from separate observations. The goal 3 will benefit from simultaneous L-band observations of the planetary systems and another LBTI survey called LEECH (LBTI Exozodi Exoplanet Common Hunt, see companion paper by A. Skemer).

\section{References}

Absil, O., et al., 2013, A\&A A, 555, 104

Bracewell, R. N., 1978, Nature, 274, 780.

Martin, S., et al., 2012, AO, 51, 3907-3921.

Mennesson, B., et al., 2011, ApJ, 243, 178.

Millan-Gabet, R., et al., 2011, ApJ, 734, 67.

Roberge, A., et al., 2012, PASP, 124, 799-808.

Stock, N. D., et al., 2010, ApJ, 724, 1238-1255. 